\title{
1 Mechanism of dominance of the Breit interaction in dielectronic recombination
}

\author{
Xiao-Min Tong调, Zhimin $\mathbf{H u}^{2,3,4}$, Yueming $\mathbf{L i}^{5}$, Xiaoyin \\ $\mathrm{Han}^{5}$, Daiji Kato ${ }^{6}$, Hirofumi Watanabe ${ }^{7}$, and Nobuyuki \\ Nakamura $^{2}$ \\ ${ }^{1}$ Graduate School of Pure and Applied Sciences, and Center for Computational \\ Sciences, University of Tsukuba, 1-1-1 Tennodai, Tsukuba, Ibaraki 305-8573, \\ Japan \\ ${ }^{2}$ Institute for Laser Science, The University of Electro-Communications, Chofu, \\ Tokyo 182-8585, Japan \\ ${ }^{3}$ Research Center of Laser Fusion, China Academy of Engineering Physics, \\ Mianyang 621900, China \\ ${ }^{4}$ Physics Institute, Heidelberg University, Im Neuenheimer Feld 226, 69120 \\ Heidelberg, Germany \\ ${ }^{5}$ Institute of Applied Physics and Computational Mathematics, P. O. Box 8009, \\ Beijing 100088, China \\ ${ }^{6}$ Department of Fusion Science, The Graduate University for Advanced Studies, \\ Sokendai, Toki, Gifu 509-5292, Japan \\ 7 Center of Applied Superconductivity and Sustainable Energy Research, Chubu \\ University, Kasugai, Aichi 487-8501, Japan
}

\begin{abstract}
The recent theoretical and experimental studies show that the Breit interaction plays a dominant role in the dielectronic recombination for some particular transitions. The detailed mechanism of why the Breit interaction is dominant for such a process is still unknown. In this work, we performed a simulation and decomposed each individual term in the transition matrix level and found that the Breit interaction is dominant when the leading term $\left(1 / r_{>}\right.$with $r>$ the larger of $r_{1}$ and $r_{2}$ ) contribution of the two-electron Coulomb interaction is vanished. Based on this mechanism, we explained why the dielectronic capture strength to $1 s 2 s^{2} 2 p_{1 / 2} J_{d}=1$ state is much stronger than the one to $1 s 2 s 2 p_{1 / 2}^{2} J_{d}=1$ as well as why the Breit interaction plays a dominant role in the anisotropic parameters. Furthermore, the present finding may guide us to search some physical processes in which the Breit interaction is dominant by simply analyzing the coupling coefficients for a given isoelectronic sequence.
\end{abstract}

Keywords: Breit interaction, Coulomb interaction, dielectronic recombination, anisotropic parameter 
Mechanism of dominance of the Breit interaction...

\section{Introduction}

Since the discovery of the Breit interaction 1, 2, its contributions to the atomic structure have been studied extensively and systematically with various theoretical methods [3, 4, 5], also as reviewed by Grant [6]. Although the Breit interaction is getting important for high- $Z$ atomic ions, its contribution to the total energy is still much smaller. Namely for nobelium $(\mathrm{Z}=102)$, the Breit contribution to the total energy is just of the order of $10^{-3}$ [7]. For total energy, the dominant contributor is the electron-nucleus Coulomb interaction, then the electron-electron Coulomb and Breit interactions. Thus, in most cases, the Breit interaction is treated perturbatively or simply ignored even for high- $\mathrm{Z}$ atoms $[8$. The Breit interaction does not only contribute to the total energy but also to dynamical processes involving two-electron interactions, like Auger decay or its inverse process dielectronic capture (DC). For such a dynamical process, the Breit interaction directly competes with the electronelectron Coulomb interaction and in most cases, the Breit interaction may modify the process quantitatively not qualitatively. The Breit interaction plays an important role for high energy electrons colliding with a highly charged ion [9, 10, 11] as well.

The effect of the Breit interaction on dielectronic recombination (DR) has been studied in highly charged ions and it was found experimentally [12] that the Breit interaction is comparable to the Coulomb interaction in lithium-like ions [12] for the autoionization state $1 s 2 s^{2} 2 p_{1 / 2} J_{d}=1$. Although the Breit interaction is important in such a DR process, the conclusion - the DC strength to $1 s 2 s^{2} 2 p_{1 / 2} J_{d}=1$ state is much stronger than the one to $1 s 2 s 2 p_{1 / 2}^{2} J_{d}=1$ state either with or without taking the Breit interaction into account - is still hold.

Later on, Fritzsche et al., 13. found that the Breit interaction may change the angular distribution of the emitted X-ray in the lithium-like DR process involving the autoionization state $1 s 2 s^{2} 2 p_{1 / 2} J_{d}=1$ in a theoretical study. For example, for lithiumLike $\mathrm{Au}$ ions, without the Breit interaction, the emitted X-ray is mainly along the electron beam direction while with the Breit interaction, the emitted X-ray is mainly along the perpendicular direction to the electron beam. This theoretical prediction was confirmed by experiment [14.

Now the question is why the Breit interaction plays such a dominant role in these processes or under what conditions the Breit interaction could be important. To answer these questions, we performed a systematic study on the DR processes involving the captures to $1 s 2 s^{2} 2 p_{1 / 2} J_{d}=1$ and $1 s 2 s 2 p_{1 / 2}^{2} J_{d}=1$ two states. Instead of just analyzing the DR rates and anisotropic parameters obtained in the simulation, we also analyzed each term of the transition matrix elements and found that the Breit interaction is important when the leading term $\left(1 / r_{>}\right.$with $r_{>}$the larger of $r_{1}$ and $r_{2}$ where $\mathbf{r}_{1}$ and $\mathbf{r}_{2}$ are the space coordinates of electron 1 and electron 2 involved in the DR transitions) contribution of the two-electron Coulomb interaction is vanished. We call this leading term the Coulomb monopole. Based on the mechanism, we can explain the experimental observations where the Breit interaction is important for both the DR rate and the anisotropic parameter.

\section{Theory}

Theory on atomic dielectronic recombination can be found in many literatures 15, 16, 17, 18, and the DR rates can be calculated by commonly used atomic packages, like, GRASP (a general-purpose relativistic atomic structure package) [5]. Here we 
only present the necessary equations which will be used for the discussion in this paper. Atomic units $\hbar=m_{e}=e=1$ are used throughout unless otherwise stated.

Dielectronic recombination involves two steps: a free electron is captured by an atomic ion with exciting another inner-shell electron to an excited state and forming an autoionization state, followed by the radiative stabilization (RS) by emitting a photon from the autoionization state. The first step is an inverse process of Auger decay. The Auger decay rate can be expressed as

$$
R_{A}=2 \pi \sum_{l j}\left|\left\langle\Psi_{J_{d}}|| V \| \Psi_{J_{i}} \psi_{l j}\right\rangle\right|^{2}
$$

with $\Psi_{J_{i}}$ the wave function of the initial state for the N-electron system, $\Psi_{J_{d}}$ the wave function of the autoionization state of the $(\mathrm{N}+1)$-electron system and $\psi_{l j}$ the continue electron wave function with angular moment $l$ and total angular momentum $j . V$ is the two-electron interaction, which includes the electron-electron Coulomb and Breit interactions. The autoionization state (or intermediate state) $\Psi_{J_{d}}$ can decay to a final state $\Psi_{J_{f}}$ radiatively by emitting a photon. $J_{i}, J_{d}, J_{f}$ are the total angular momenta of the initial, intermediate and final states, respectively. The angular distribution of the emitted photon is written as

$$
\frac{d \sigma}{d \Omega}=\frac{\sigma_{T}}{4 \pi}\left(1+\beta P_{2}(\cos \theta)\right),
$$

with $\sigma_{T}$ the total DR cross section, $\theta$ the angle between the emitted photon and the electron beam directions, and $P_{2}(\cos \theta)$ the second order Legendre polynomial. Note here we focus on the dipole transition and the general expression for multipole transitions can be found in Refs. [19, 20]. The anisotropic parameter $\beta$ is given by

$$
\beta=(-1)^{1+J_{d}+J_{f}}\left[\frac{3\left(2 J_{d}+1\right)}{2}\right]^{1 / 2}\left\{\begin{array}{ccc}
1 & 1 & 2 \\
J_{d} & J_{d} & J_{f}
\end{array}\right\} \frac{P_{J_{i} J_{d}}^{(2)}}{P_{J_{i} J_{d}}^{(0)}}
$$

with

$$
\begin{aligned}
P_{J_{i} J_{d}}^{(L)}= & \frac{(-1)^{J_{i}+L+j_{d}-1 / 2}}{2\left(2 J_{i}+1\right)} \sum_{l j l^{\prime} j^{\prime}} i^{l-l^{\prime}}\left[j, j^{\prime}, l, l^{\prime}, L\right]^{1 / 2}\left[\begin{array}{ccc}
l & l^{\prime} & L \\
0 & 0 & 0
\end{array}\right]\left\{\begin{array}{ccc}
j^{\prime} & j & L \\
l & l^{\prime} & 1 / 2
\end{array}\right\} \\
& \times\left\{\begin{array}{ccc}
J_{d} & J_{d} & L \\
j & j^{\prime} & J_{i}
\end{array}\right\}\left\langle\Psi_{J_{d}}\|V\| \Psi_{J_{i}} \psi_{l j}\right\rangle\left\langle\Psi_{J_{i}} \psi_{l^{\prime} j^{\prime}}\|V\| \Psi_{J_{d}}\right\rangle,
\end{aligned}
$$

where the Wigner $3 j, 6 j$ symbols are used. Here we focus on two transition lines of lithium-like isoelectronic ions. They are

$$
\begin{aligned}
& \text { Line a: }\left|1 s^{2} 2 s \psi_{l j}\right\rangle \stackrel{\mathrm{DC}}{\longrightarrow}\left|1 s 2 s^{2} 2 p_{1 / 2}\left(J_{d}=1\right)\right\rangle \stackrel{\mathrm{RS}}{\longrightarrow}\left|1 s^{2} 2 s^{2}\left(J_{f}=0\right)\right\rangle, \\
& \text { Line b: }\left|1 s^{2} 2 s \psi_{l j}\right\rangle \stackrel{\mathrm{DC}}{\longrightarrow}\left|1 s 2 s 2 p_{1 / 2}^{2}\left(J_{d}=1\right)\right\rangle \stackrel{\mathrm{RS}}{\longrightarrow}\left|1 s^{2} 2 s 2 p_{1 / 2}\left(J_{f}=0,1\right)\right\rangle .
\end{aligned}
$$

For Line $\mathrm{b}$, due to the selection rule, the initial continue state can only be an $s$ partial wave and thus the anisotropic parameter $\beta$ is zero. For Line a, the anisotropic parameter can be explicitly expressed as

$$
\beta=-\frac{1}{2} \frac{\left(2 \sqrt{2} V_{1}+V_{2}\right) \cdot V_{2}}{V_{1}^{2}+V_{2}^{2}},
$$

with

$$
\begin{aligned}
& V_{1}=V_{p_{1 / 2}}=\left\langle 1 s 2 s^{2} 2 p_{1 / 2}\left(J_{d}=1\right)\left\|V_{C}+V_{\text {Breit }}\right\| 1 s^{2} 2 s \epsilon p_{1 / 2}\right\rangle=V_{1}^{C}+V_{1}^{B}(6) \\
& V_{2}=V_{p_{3 / 2}}=\left\langle 1 s 2 s^{2} 2 p_{1 / 2}\left(J_{d}=1\right)\left\|V_{C}+V_{\text {Breit }}\right\| 1 s^{2} 2 s \epsilon p_{3 / 2}\right\rangle=V_{2}^{C}+V_{2}^{B}(.7)
\end{aligned}
$$




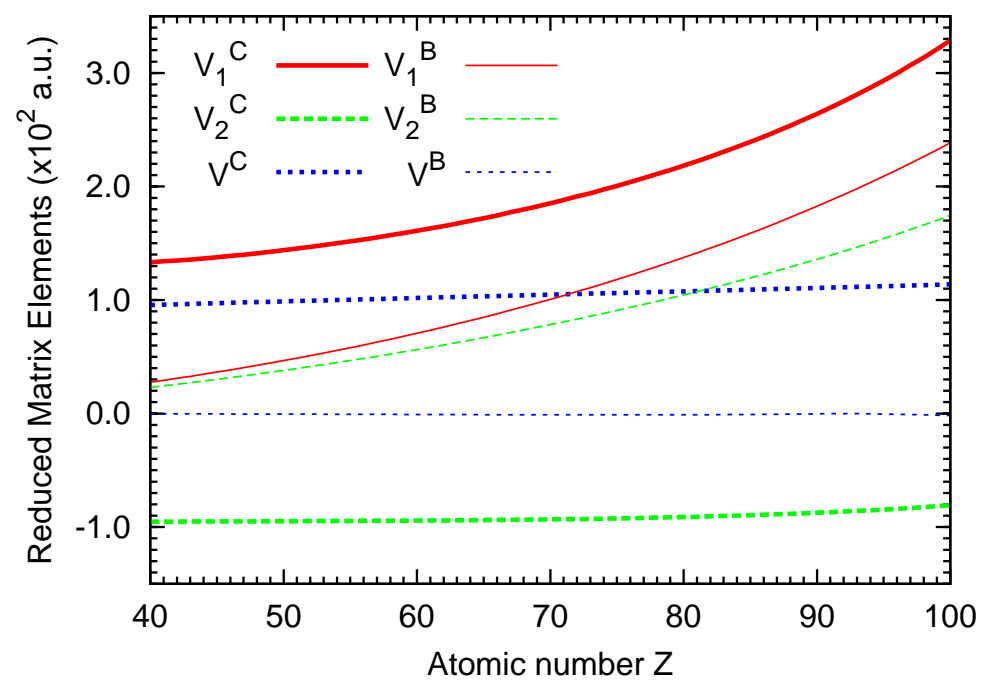

Figure 1. (Color online) Reduced matrix elements of dielectronic capture for Lines a and b. $V_{1}^{C}$ (thick solid line), $V_{2}^{C}$ (thick dashed line) and $V^{C}$ (thick dotted line) are defined in Eqs. 67- 8) with the Coulomb interaction only. $V_{1}^{B}$ (thin solid line), $V_{2}^{B}$ (thin dashed line) and $V^{B}$ (thin dotted line) are defined in Eqs. 67-8 with the generalized Breit interaction only.

Here $V_{C}, V_{B r e i t}$ are the electron-electron Coulomb and Breit interactions. Note that the anisotropic parameter depends on the DC process so we will analyze the DC transition matrix $V_{1}, V_{2}$ in detail. For the convenience of discussion, we also give the reduced transition matrix element for Line $b$ as

$$
V=V_{s_{1 / 2}}=\left\langle 1 s 2 s 2 p_{1 / 2}^{2}\left(J_{d}=1\right)\left\|V_{C}+V_{B r e i t}\right\| 1 s^{2} 2 s \epsilon s_{1 / 2}\right\rangle=V^{C}+V^{B} .
$$

\section{Results and discussion}

We first calculated the single-electron wave function from the relativistic density functional theory with the self-interaction correction 21. and then calculated the reduced matrix elements. The coupling coefficients were calculated by using the ANCO package [22] and the detailed numerical method can be found in Ref. 23. The reduced matrix elements were calculated using the single configuration approximation as well as configuration interactions and the two results are close to each other within two or three digits. Therefore to simplify the discussion, we only present the results from the single configuration simulation. We also present the results from the generalized Breit interaction (GBI) 24] and the Breit interaction (BI0) in the zero-frequency limit. To study lithium-like atomic ions systematically, we calculated all the ions with $Z=40-100$. Note that to calculate the reduced matrix element, there is an arbitrary phase factor and we chose the phase factor in such a way that $V_{1}^{C}$ is positive.

\subsection{Reduced matrix elements for dielectronic capture}

Figure 1 shows the reduced matrix elements for the Coulomb interaction only and the GBI defined in Eqs. 6)-(8). From a scaling law, the Coulomb interaction is scaled by atomic number $\mathrm{Z}$ and the GBI is scaled by $\left(Z^{3} / c^{2}\right)$ with $c$ the velocity of light. Indeed, $V_{1}^{C}$ is the largest one among the transition matrix elements and it increases monotonically as $\mathrm{Z}$ increases. $V_{1}^{B}$ increases rapidly and is comparable but still smaller than $V_{1}^{C}$ even for very 
Table 1. Coupling coefficients of the reduced matrix elements of dielectronic captures for Lines a and $b$.

\begin{tabular}{|c|c|c||c|c|c|}
\hline Term & $k$ & $X^{k}$ & Term & $k$ & $X^{k}$ \\
\hline$V_{1}:\left(2 s 2 p_{1 / 2} \mid 1 s \epsilon p_{1 / 2}\right)$ & 0 & $-1 / 2$ & $V_{2}:\left(2 s 2 p_{1 / 2} \mid 1 s \epsilon p_{3 / 2}\right)$ & 1 & $-1 / 3$ \\
$V_{1}:\left(2 s 2 p_{1 / 2} \mid 1 s \epsilon p_{1 / 2}\right)$ & 1 & $-1 / 6$ & $V_{2}:\left(2 s 2 p_{1 / 2} \mid \epsilon p_{3 / 2} 1 s\right)$ & 1 & $1 / 3$ \\
$V_{1}:\left(2 s 2 p_{1 / 2} \mid \epsilon p_{1 / 2} 1 s\right)$ & 1 & $-1 / 3$ & & & \\
\hline$V:\left(2 p_{1 / 2}^{2} \mid 1 s \epsilon s\right)$ & 0 & $1 / 2$ & $V:\left(2 p_{1 / 2}^{2} \mid 1 s \epsilon s\right)$ & 1 & $1 / 2$ \\
\hline
\end{tabular}

high $\mathrm{Z}$ ions. This means that for the DC strength, the Coulomb interaction is still a dominant contributor for Line a. $V_{2}^{B}$ follows the trend of $V_{1}^{B}$ but smaller than $V_{1}^{B}$ and $V^{B}$ is almost zero. Interestingly, $V_{2}^{C}, V^{C}$ are almost constants and smaller than $V_{1}^{C}$. Especially for $V_{2}^{C}$, it has an opposite sign with $V_{2}^{B}$. The absolute value of $V_{2}^{B}$ is smaller than $V_{2}^{C}$ for low $\mathrm{Z}$ atomic ions and reaches the same magnitude at $Z=73$, then is larger than $V_{2}^{C}$ for high- $Z$ atomic ions. Overall, from the reduced transition matrix elements, we see that the Coulomb interaction is a dominant contributor to the DC process and the GBI contribution is comparable but still smaller than the one of the Coulomb interaction. The key step to understand why the Breit interaction plays a dominant role for anisotropic parameters is to understand why $V_{2}^{C}$ is smaller.

Both the Coulomb and Breit interactions are two-elecron operators and they can be evaluated as a product of coupling coefficients and radial integrals as

$$
\begin{aligned}
& \left\langle\Psi_{J_{d}}|| V_{C} \| \Psi_{J_{i}} \phi_{l j}\right\rangle=\sum_{k} X^{k} \cdot R_{C}^{k}, \\
& \left\langle\Psi_{J_{d}}|| V_{\text {Breit }}|| \Psi_{J_{i}} \phi_{l j}\right\rangle=\sum_{k} X^{k} \cdot R_{B}^{k},
\end{aligned}
$$

where $X^{k}$ is the coupling coefficient which is independent from the atomic number $\mathrm{Z}$ and $R_{C}^{k}, R_{B}^{k}$ are the radial integrals for an irreducible tensor operator of rank $k$. Note that we followed the convention of the ANCO program [22] and the coefficients are listed in Table 1 . For the Coulomb interaction, $R_{C}^{k}$ is decided by the integration of the operator

$$
\begin{aligned}
V_{C} & =\frac{1}{\left|\mathbf{r}_{1}-\mathbf{r}_{2}\right|}=\sum_{k}\left(\frac{r_{<}}{r_{>}}\right)^{k} \frac{1}{r_{>}} P_{k}(\cos \gamma) \\
& =\sum_{k}\left(\frac{r_{<}}{r_{>}}\right)^{k} \frac{1}{r_{>}} \frac{4 \pi}{2 k+1} \sum_{m} Y_{-m}^{k}\left(\hat{\mathbf{r}}_{1}\right) Y_{m}^{k}\left(\hat{\mathbf{r}}_{2}\right),
\end{aligned}
$$

where $r_{<}\left(r_{>}\right)$stands for the smaller (larger) one of $\left|\mathbf{r}_{1}\right|,\left|\mathbf{r}_{2}\right|, P_{k}$ the $k$-order of Legendre polynomials, $\gamma$ the angle between $\mathbf{r}_{1}, \mathbf{r}_{2}$, and $Y^{k}$ the k-order of spherical harmonics. $R_{C}^{k}$ can be written as

$$
R_{C}^{k} \propto<a|| Y^{k}|| c><b|| Y^{k} \| d>,
$$

where $a, b, c, d$ are the four orbits involved in the two-electron operator as $(a b \mid c d)$ in Table 1 . For example, the Coulomb contribution of $V_{2}$ can be evaluated as

$$
\begin{aligned}
V_{2}^{C} \propto & -\frac{1}{3}\left\langle 2 s \| Y^{k=1}|| 1 s\right\rangle\left\langle 2 p_{1 / 2}\left\|Y^{k=1}\right\| \epsilon p_{3 / 2}\right\rangle \\
& +\frac{1}{3}\left\langle 2 s \| Y^{k=1}|| \epsilon p_{3 / 2}\right\rangle\left\langle 2 p_{1 / 2} \| Y^{k=1}|| \epsilon 1 s\right\rangle \\
= & \frac{1}{3}\left\langle 2 s\left\|Y^{k=1}\right\| \epsilon p_{3 / 2}\right\rangle\left\langle 2 p_{1 / 2}\left\|Y^{k=1}\right\| \epsilon 1 s\right\rangle .
\end{aligned}
$$

The first term of right hand in Eq. 13 is zero because $\left\langle 2 s\left\|Y^{k=1}\right\| 1 s\right\rangle=0$. 
For the generalized Breit interaction [24], $R_{\mathrm{B}}^{k}$ is decided by the integration of the operator

$$
V_{\text {Breit }}=-\boldsymbol{\alpha}_{1} \cdot \boldsymbol{\alpha}_{2} \frac{\cos (\omega r)}{r}+\left(\boldsymbol{\alpha}_{1} \cdot \nabla_{1}\right)\left(\boldsymbol{\alpha}_{2} \cdot \nabla_{2}\right) \frac{\cos (\omega r)-1}{\omega^{2} r},
$$

where $r=\left|\mathbf{r}_{1}-\mathbf{r}_{2}\right|$ and $\omega$ is the virtual photon energy divided by $c$, and $\boldsymbol{\alpha}$ the Dirac matrixes. If we choose $\omega=0$, we get the Breit interaction (BI0) in the zero-frequency limit. Similar to $R_{C}^{k}, R_{B}^{k}$ can be evaluated as

$$
R_{B}^{k} \propto<a\left\|T^{k}\right\| c><b\left\|T^{k}\right\| d>,
$$

with $T^{k}$ the tensor of rank $k$. The general expression of $T^{k}$ is complicate and can be found in Ref. [3] for details. The tensor operator of the first term of right hand in Eq. [15 can be written

$$
T^{k}=\sum_{k^{\prime}} \boldsymbol{\alpha} Y^{k^{\prime}}
$$

as a vector spherical harmonics of order of $k$ 25. For example, the Breit contribution to $V_{2}$ involves the tensor product of $\boldsymbol{\alpha}$ (tensor of rank 1) with the space tensor $Y^{k^{\prime}}$ (spherical harmonics) and it dose not vanish for $Y^{k^{\prime}=0}$. This should be the largest one for the Breit interaction.

Generally speaking, for Coulomb interaction, the high order $(k)$ multipole contributions are getting smaller because $\left(r_{<} / r_{>}\right)^{k}$ decreases as $k$ increases. For $V_{1}^{C}$, the monopole $(k=0)$ contribution is not vanished and it is the major contributor to the DC transition matrix elements as shown in Table 1. For $V_{2}^{C}$, the monopole is vanished and this results in smaller value of $V_{2}^{C}$. Although the coupling coefficient of $V$ is not zero for $k=0$, the reduced matrix element of the Coulomb interaction is zero because for a single electron irreducible tensor operator of rank $0, s \rightarrow p_{1 / 2}\left(\left\langle 2 p_{1 / 2}|| Y^{0} \| 1 s\right\rangle\right)$ matrix element is zero due to the parity conservation. For Breit interaction, the leading contribution should be the transition matrix elements with the tensor of rank $k=1$ with the spherical harmonic of $k^{\prime}=0$. From above analysis and the simulation data shown in Fig. 1, we conclude that the relative weak strength of Line b comparing with Line a is due to the lack of Coulomb monopole contributions.

\subsection{Ratio of the dielectronic capture strengths}

Since directly measuring the anisotropic parameters is still difficult in the Tokyo EBIT (electron beam ion trap) 26], the anisotropic parameter was extracted from the measured ratio of DC strengths of Lines a and b, and the emitted X-ray at $90^{\circ}$ to the electron beam. Therefore we first analyze the ratio of DC strengths between Lines a and b as shown in Fig. 2 . Without the Breit interaction, the ratio increases slowly from 3 at $Z=40$ to 9 at $Z=100$. With the $\mathrm{BI} 0$, the ratio increases rapidly from 3.5 at $\mathrm{Z}=40$ to 23 at $\mathrm{Z}=100$. With the GBI, the ratio is slightly higher than the one of the BI0. The results show that the Breit interaction enhanced the ratio by more than a factor of two for a very high $\mathrm{Z}$ atomic ion, while in Fig. 1 $V_{1}^{C}$ is always the largest one. The ratio enhanced by a factor of two does not mean the Breit interaction is the dominant one because the DC strength is proportional to the square of the reduced matrix elements $\left|V_{1}^{C}+V_{1}^{B}\right|^{2}$ and if $V_{1}^{B}$ is just about $50 \%$ of the $V_{1}^{C}$, the DC strength can be doubled. The results are consistent with the experiments 12, 14, 27, which showed that the Breit interaction enhances the ratio by a factor of two. Although the GBI modifies the Ratio slightly over the BI0, the ratio of the GBI is more closer to the measured ones. Discrepancies between the simulation and measurement may call further theoretical studies as well as experiments with better resolution.

\subsection{Dominance of the Breit interaction for anisotropic parameters}

The reduced matrix elements in Fig. 1 show that the Coulomb interaction is always the dominant one for the total DC strengths. For anisotropic parameters, the Breit interaction 


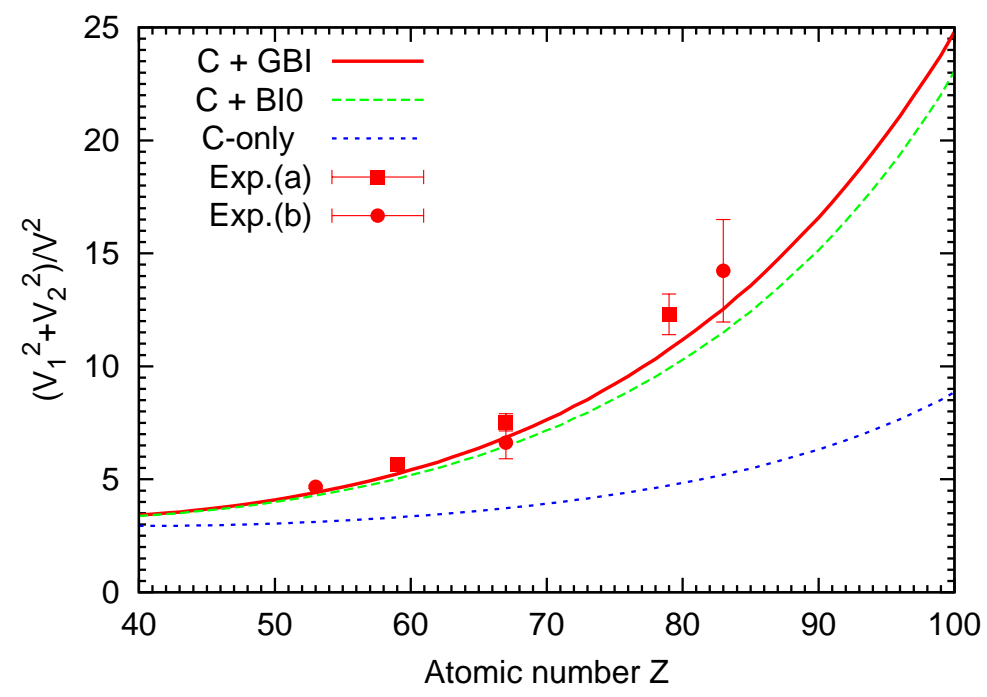

Figure 2. (Color online) Ratios of dielectronic capture strengths of Lines a and b. Solid line: the theoretical results with the generalized Breit interaction, dashed line: the theoretical results with the Breit interaction in the zero-frequency limit, and dotted line: the theoretical results without the Breit interaction. The data of Exp.(a) are from Ref. 27] and the ones of Exp.(b) are from Ref. 12].

may change the angular distribution of the emitted X-ray from peaked on the forward direction along the electron beam $(\beta>0)$ with the Coulomb interaction only to peaked on the perpendicular direction to the electron beam $(\beta<0)$ with the Breit interaction for high-Z ions as shown in Fig. 3. To understand the mechanism, we approximate the anisotropic parameter in Eq. (5) as

$$
\beta \approx-\sqrt{2} \frac{V_{2}}{V_{1}}
$$

because $V_{1}$ is positive and always larger than the value of $\left|V_{2}\right|$ as shown in Fig. 1. In the figure, we see that the Breit interaction for $V_{2}$ is comparable with the Coulomb interaction for low-Z ions and is larger than the Coulomb interaction for high $\mathrm{Z}$ ions with $\mathrm{Z}>72$. This is mainly because of the vanishing of the Coulomb monopole for $V_{2}$. For this particular transition, Line a, the anisotropic parameter is almost linearly proportional to the reduced matrix element $V_{2}$, in which the Breit interaction plays a dominant role for high $\mathrm{Z}$ ions. Our results of the Coulomb only (dash-dotted line) and Coulomb plus BI0 (dotted line) are in good agreements with the results (open circles and filled circles) from Fritzsche et al., [13. Again, we see that the BGI modifies the anisotropic parameters quantitatively not qualitatively over the BI0. Both the BI0 and GBI's results are in reasonable agreement with the available measurements 14, 27.

\section{Summary and Conclusions}

To summarize, we have investigated the mechanism of the dominance of the Breit interaction in the angular distribution of the emitted X-ray in dielectronic recombination of lithiumlike isoelectronic ions for $Z=40-100$. By analyzing the reduced matrix elements in the dielectronic capture when off-diagonal elements are involved, we found that the Breit interaction could be important when the Coulomb monopole interaction is vanished. Based on the mechanism, we have explained the anomalous Breit interaction enhanced ratio of dielectronic capture strengths for Lines $\mathrm{a}$ and $\mathrm{b}$ as well as the dominance of the Breit 


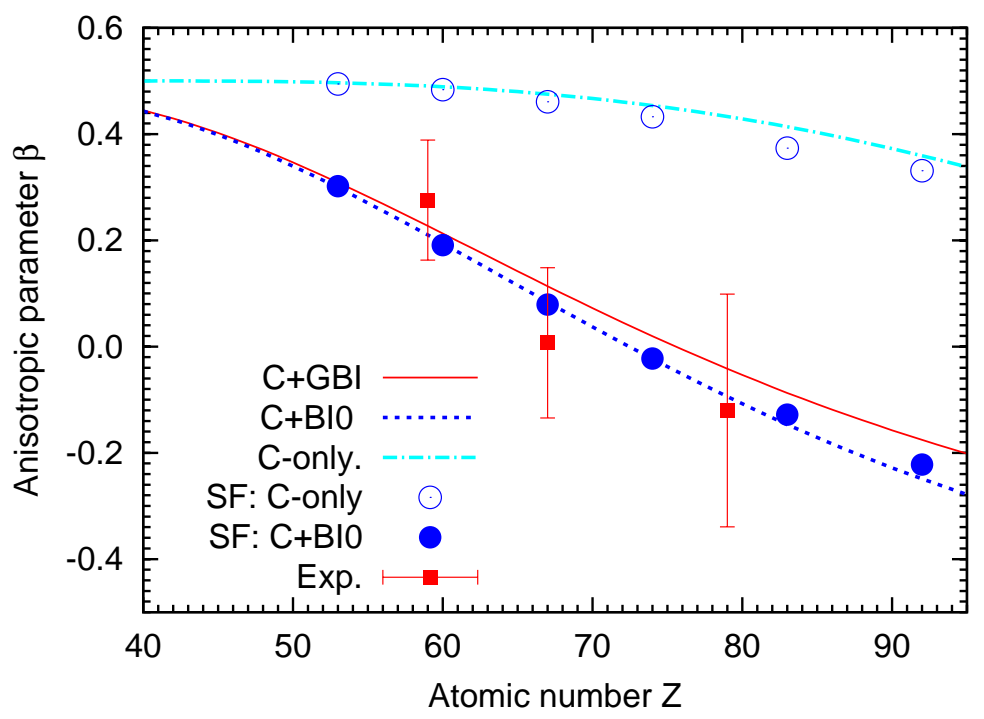

Figure 3. (Color online) Anisotropic parameters of Line a. Solid line (C+GBI): the theoretical results with the generalized Breit interaction, dashed line $(\mathrm{C}+\mathrm{BI})$ : the theoretical results with the Breit interaction in the zero-frequency limit, dashdotted line (Coulomb): the theoretical results with the Coulomb interaction only; filled and open circles: from Ref. 13 . The experimental data of $\operatorname{Pr}(Z=59)$ and $\mathrm{Ho}(Z=67)$ are from ref. 27] and the datum of $\mathrm{Au}(Z=79)$ is from ref. [14.

interaction in the angular distribution of the emitted X-ray in dielectronic recombination. By comparing the results of the generalized Breit interaction and the Breit interaction in the zero-frequency limit, we conclude that the modification of GBI over the BI0 is smaller and can be neglected in most cases. The present work may provide an effective way to search a physical process in which the Breit interaction is important simply by examing the coupling coefficients which are the same for a given isoelectronic sequence under the single configuration approximation.

\section{Acknowledments}

XMT was supported by a Grand-in-Aid for Scientific Research (C24540421) from the Japan Society for the Promotion of Science. ZH acknowledges the support of the GermanResearch Foundation (DFG) within the Emmy Noether program under Contract No. TA 740/1-1. DK was supported by JSPS-NRF-NSFC A3 Foresight Program in the field of Plasma Physics (NSFC: No.11261140328, NRF: 2012K2A2A6000443) .

\section{References}

[1] Breit G 1930 Phys. Rev. 36 383-397

[2] Breit G 1932 Phys. Rev. 39 616-624

[3] Grant I P and Pyper N C 1976 J. Phys. B: At. Mol. Phys. 9 761-774

[4] Sapirstein J 1998 Rev. Mod. Phys. 70 55-76

[5] Jonsson P, He X, Fischer C F and and I P G 2007 Comput. Phys. Commun. 177 597-622

[6] Grant I P 2010 J. Phys. B: At. Mol. Opt. Phys. 43074033

[7] Mann J B and Johnson W R 1971 Phys. Rev. A 4 41-51

[8] Karasiev V V, Ludena E V and Shukruto O A 2004 Phys. Rev. A 69052509

[9] Marrs R E, Elliott S R and Scofield J H 1997 Phys. Rev. A 56 1338-1345

[10] Sampson D H, Zhang H L and Fontes C J 2009 Phys. Rep. 477111 - 214 
[11] Gumberidze A, Thorn D B, Fontes C J, Najjari B, Zhang H L, Surzhykov A, Voitkiv A, Fritzsche S, Banaś D, Beyer H, Chen W, DuBois R D, Geyer S, Grisenti R E, Hagmann S, Hegewald M, Hess S, Kozhuharov C, Märtin R, Orban I, Petridis N, Reuschl R, Simon A, Spillmann U, Trassinelli M, Trotsenko S, Weber G, Winters D F A, Winters N, Yu D and Stöhlker T 2013 Phys. Rev. Lett. 110213201

[12] Nakamura N, Kavanagh A P, Watanabe H, Sakaue H A, Li Y, Kato D, Currell F J and Ohtani S 2008 Phys. Rev. Lett. 100073203

[13] Fritzsche S, Surzhykov A and Stöhlker T 2009 Phys. Rev. Lett. 103113001

[14] Hu Z, Han X, Li Y, Kato D, Tong X and Nakamura N 2012 Phys. Rev. Lett. 108(7) 073002 URL http://link.aps.org/doi/10.1103/PhysRevLett.108.073002

[15] Inal M K and Dubau J 1987 J. Phys. B: At. Mol. Phys. 204221

[16] Chen M H, Reed K J, Guo D S and Savin D W 1998 Phys. Rev. A 58 4539-4547

[17] Qu Y Z, Wang J G, Yuan J K and Li J M 1998 Phys. Rev. A 57 1033-1037

[18] Fritzsche S, Kabachnik N M and Surzhykov A 2008 Phys. Rev. A 78032703

[19] Berezhko E G and Kabachnik N M 1977 J. Phys. B: At. Mol. Phys. 102467

[20] Tseng H K, Pratt R H, Yu S and Ron A 1978 Phys. Rev. A 17 1061-1079

[21] Tong X M and Chu S I 1998 Phys. Rev. A 57(1) 452-461 URL http://link.aps.org/doi/10. 1103/PhysRevA.57.452

[22] Gaigalas G and Fritzsche S 2002 Comput. Phys. Commun. 148 349-351

[23] Tong X M, Nakamura N, Ohtani S, Watanabe T and Toshima N 2009 Phys. Rev. A 80(4) 042502 URL http://link.aps.org/doi/10.1103/PhysRevA.80.042502

[24] Grant I P and McKenzie B J 1980 J. Phys. B: At. Mol. Phys. 13 2671-2681

[25] Tong X M, Li J M, Kissel L and Pratt R H 1990 Phys. Rev. A 42(3) 1442-1449 URL http://link.aps.org/doi/10.1103/PhysRevA.42.1442

[26] Nakamura N, Asada J, Currell F J, Fukami T, Hirayama T, Motohashi K, Nagata T, Nojikawa E, Ohtani S, Okazaki K, Sakurai M, Shiraishi H, Tsurubuchi S and Watanabe H 1997 Phys. Scr. T. 73 362-364

[27] Hu Z, Li Y, Han X, Kato D, Tong X, Watanabe H and Nakamura N 2014 Phys. Rev. A 90 062702 